\title{
EXTENSIONS OF MONOTONE OPERATOR FUNCTIONS
}

\author{
J. D. CHANDLER, JR. ${ }^{1}$
}

\begin{abstract}
It is shown that a monotone operator function $f$ defined on an open subset $\Delta$ of the real numbers may be extended to a monotone operator function on the convex hull of $\Delta$.
\end{abstract}

Let $f$ be a bounded real-valued Borel-measurable function defined on a Borel subset $\Delta$ of the real numbers. Let $A$ be a bounded selfadjoint operator on a separable complex Hilbert space $H$ such that the spectrum $\sigma(A)$ of $A$ is contained in $\Delta$. Then $f(A)$ is the selfadjoint operator on $H$ defined by

$$
\langle f(A) \phi, \psi\rangle=\int_{\sigma(A)} f(\lambda)\langle E(d \lambda) \phi, \psi\rangle, \quad \phi, \psi \in H,
$$

where $E$ is the resolution of the identity corresponding to $A$.

The function $f$ is said to be a monotone operator function on $\Delta$ if $f(A) \leq f(B)$ whenever $A$ and $B$ are bounded selfadjoint operators on a Hilbert space $H$ such that $\sigma(A) \subseteq \Delta, \sigma(B) \subseteq \Delta$, and $A \leq B$. If $f$ satisfies this monotonicity condition for the totality of finite-dimensional complex Hiltert spaces, then $f$ is said to be a monotone matrix function on $\Delta$.

The following characterization of monotone operator functions is essentially due to Loewner [5]. Loewner proved the theorem for monotone matrix functions; Bendat and Sherman [1] proved that a function $f$ is monotone matrix on an open interval $(a, b)$ if and only if $f$ is monotone operator on $(a, b)$.

THEOREM 1. A real-valued function $f$ defined on an open interval $(a, b)$ is a monotone operator function on $(a, b)$ if and only if $f$ is analytic on $(a, b)$, can be analytically continued onto the upper half-plane, and represents there a holomorphic function with nonnegative imaginary 1 rt.

It will be shown that there is a similar characterization of monotone operator functions defined on an arbitrary open subset $\Delta$ of the real numbers.

Let $f$ be a monotone operator function on an open subset $\Delta$ of the real numbers. By Theorem $1, f$ is continuously differentiable on $\Delta$; see also [4, p. 73]. Associated with $f$ is the kernel $K$, defined on $\Delta \times \Delta$ by

$$
K(\lambda, \mu)=\frac{f(\lambda)-f(\mu)}{\lambda-\mu}(\lambda \neq \mu), \quad K(\lambda, \lambda)=f^{\prime}(\lambda) .
$$

Loewner made extensive use of this kernel in his study of monotone matrix

Received by the editors May 21, 1975.

AMS (MOS) subject classifications (1970). Primary 47A60, 47B15; Secondary 30A14, 30A76. Key words and phrases. Monotone operator functions, functions with positive imaginary part.

${ }^{1}$ Research supported by NSF Grant GP-31483XZ. 
functions. The following theorem is essentially due to Loewner [5]; see also [1] and [4].

THEOREM 2. Let $f$ be a monotone operator function on an open subset $\Delta$ of the real numbers. Then $K$ is a positive matrix; that is,

$$
\sum_{j=1}^{n} \sum_{k=1}^{n} K\left(\lambda_{j}, \lambda_{k}\right) c_{j} \bar{c}_{k} \geq 0
$$

whenever $\lambda_{1}, \ldots, \lambda_{n}$ belong to $\Delta$ and $c_{1}, \ldots, c_{n}$ are complex numbers, for $n=1,2,3, \ldots$.

Proof. Let $A$ and $B$ be self adjoint operators on an $n$-dimensional Hilbert space $H$, and let $A$ and $B$ have spectral representations

$$
A=\sum_{j=1}^{n} \lambda_{j} E_{j}, \quad B=\sum_{k=1}^{n} \mu_{k} F_{k},
$$

where $\lambda_{1}, \ldots, \lambda_{n}$ and $\mu_{1}, \ldots, \mu_{n}$ belong to $\Delta$. An easy computation shows that

$$
f(B)-f(A)=\sum_{j=1}^{n} \sum_{k=1}^{n} K\left(\lambda_{j}, \mu_{k}\right) E_{j}(B-A) F_{k} .
$$

Let $B=A+\epsilon P$ be a one-dimensional perturbation of $A$, say $P=\langle\cdot, \phi\rangle \phi$ for some $\phi$ in $H$, such that $\epsilon>0$ and $\sigma(A+\epsilon P) \subseteq \Delta$. Then $B$ has a spectral representation

$$
B=\sum_{k=1}^{n} \lambda_{k}(\epsilon) E_{k}(\epsilon)
$$

where $\lambda_{k}(\epsilon) \rightarrow \lambda_{k}$ as $\epsilon \downarrow 0(k=1, \ldots, n)$. Equation (2) shows that, for any $\psi$ in $H$,

$$
\begin{aligned}
0 & \leq\langle(1 / \epsilon)[f(B)-f(A)] \psi, \psi\rangle \\
& =\sum_{j=1}^{n} \sum_{k=1}^{n} K\left(\lambda_{j}(\epsilon), \lambda_{k}\right)\left\langle E_{j} P E_{k}(\epsilon) \psi, \psi\right\rangle ;
\end{aligned}
$$

take $\epsilon \downarrow 0$ to show that

$$
\sum_{j=1}^{n} \sum_{k=1}^{n} K\left(\lambda_{j}, \lambda_{k}\right)\left\langle E_{k} \psi, \phi\right\rangle\left\langle\phi, E_{j} \psi\right\rangle \geq 0 .
$$

Now choose $\phi=\sum_{j=1}^{n} c_{j} e_{j}$ and $\psi=\sum_{k=1}^{n} e_{k}$, where $E_{j} e_{j}=e_{j}$ and $\left\|e_{j}\right\|$ $=1(j=1, \ldots, n)$, to show that

$$
\sum_{j=1}^{n} \sum_{k=1}^{n} K\left(\lambda_{j}, \lambda_{k}\right) c_{j} \bar{c}_{k} \geq 0 .
$$

Loewner [5] proved that the converse of Theorem 2 is true if $\Delta$ is an open interval $(a, b)$. This result, together with Theorem 1 , shows that a real-valued continuously differentiable function $f$, defined on an open interval $(a, b)$, admits an analytic continuation $F$ onto the upper half-plane $\Pi^{+}$with 
$\operatorname{Im}(F) \geq 0$ on $\Pi^{+}$, if and only if the kernel $K$ associated with $f$ is a positive matrix.

This aspect of Loewner's work was generalized by Rosenblum and Rovnyak [6, Theorem 8]; the following theorem is an immediate consequence of their generalization.

THEOREM 3. Let $f$ be a real-valued continuously differentiable function on an open subset $\Delta$ of the real numbers, and let $K$ be the kernel associated with $f$, as in (1). If $K$ is a positive matrix, then there exists a function $F$ such that

(i) $F$ is separately holomorphic on the upper and lower half-planes,

(ii) $F$ has nonnegative imaginary part on the upper half-plane, and

(iii) $F$ may be analytically continued by reflection across $\Delta$ so that

$$
f(x)=F(x+i 0)=F(x-i 0), \quad x \in \Delta .
$$

The next theorem is due to Šmul'jan [7]; it depends heavily on the work of Davis [2], [3] on convex operator functions.

THEOREM 4. Let $f$ be a monotone operator function on $\{a\} \cup(b, c)$, where $-\infty<a<b<c<\infty$. Then there exists a unique monotone operator function $g$ defined on $(a, c)$ such that $g=f$ on $(b, c)$ and $g(a+0) \geq f(a)$.

Śmul'jan proved this theorem for the special case $a=0, b=1$, and $c=2$. As noted in [7], the general case follows by considering $f \circ h$, where $h$ is the unique linear fractional transformation such that $h(0)=a, h(1)=b$, and $h(2)=c$.

Theorems 3 and 4 are now combined to yield a characterization of monotone operator functions defined on arbitrary open subsets of the real numbers.

THEOREM 5. Let $f$ be a monotone operator function defined on an open subset $\Delta$ of the real numbers. Then there exists a monotone operator function $h$ defined on the convex hull $D$ of $\Delta$ such that $f=h$ on $\Delta$.

Proof. If $f$ is a monotone operator function on $\Delta$, then $f$ is continuously differentiable on $\Delta$. By Theorem 2, the kernel $K$ associated with $f$ is a positive matrix. By Theorem 3, $f$ admits an analytic continuation $F$ onto the upper half-plane that satisfies (i) -(iii) of that theorem.

Let $a$ and $c$ be arbitrary points in $\Delta$ with $a<c$. Since $\Delta$ is open, there is a point $b$ in $\Delta$ such that $a<b<c$ and $(b, c) \subseteq \Delta$. By Theorem 4 , there exists a monotone operator function $g$ on $(a, c)$ such that $f=g$ on $(b, c)$. By Theorem $3, g$ admits an analytic continuation $G$ onto the upper half-plane that satisfies (i) -(iii) of that theorem, with $\Delta$ replaced by $(a, c)$.

Let $F$ and $G$ be analytically continued by reflection across $\Delta$ and $(a, c)$, respectively, as in Theorem 3(iii). But $F=f$ on $\Delta, G=g$ on $(a, c)$, and $f=g$ on $(b, c)$; since the holomorphic functions $F$ and $G$ coincide on $(b, c)$, they coincide on the intersection of their domains. Thus $F=G$ on $\Pi^{+}$, and so $F$ may be analytically continued across $\Delta \cup(a, c)$ by reflection. Since $a$ and $c$ are arbitrary points in $\Delta$, it follows that $F$ may be analytically continued across the convex hull $D$ of $\Delta$ by reflection. 
Let $H$ be this extension of $F$ to the complex plane slit along $(-\infty, \infty)-D$, and let $h$ be the restriction of $H$ to $D$. Since $H$ has nonnegative imaginary part in the upper half-plane and since $h$ is real-valued and analytic on $D, h$ is a monotone operator function on $D$, by Theorem 1 . Since $h=f$ on $\Delta, h$ is the desired extension of $f$ to $D$.

The converse of Theorem 5 follows directly from the definition of "monotone operator function": if $g$ is a monotone operator function on an interval $D$ and if $\Delta$ is an open subset of $D$, then $g \mid \Delta$ is a monotone operator function on $\Delta$.

Note that Theorem 3 was essential to the proof of Theorem 5; Loewner's original theorem (Theorem 1 above) does not provide the necessary single analytic continuation of $f$ onto the upper half-plane.

The author is grateful to Professors Marvin Rosenblum and James Rovnyak for suggesting this problem.

\section{REFERENCES}

1. J. Bendat and S. Sherman, Monotone and convex operator functions, Trans. Amer. Math. Soc. 79 (1955), 58-71. MR 18, 588.

2. C. Davis, A Schwarz inequality for convex operator functions, Proc. Amer. Math. Soc. 8 (1957), 42-44. MR 18, 812.

3. - Notions generalizing convexity for functions defined on spaces of matrices, Proc. Sympos. Pure Math., vol. 7, Amer. Math. Soc., Providence, R.I., 1963, pp. 187-201. MR 27 \#5771.

4. W. Donoghue, Monotone matrix functions and analytic continuation, Springer-Verlag, New York, Heidelberg, Berlin, 1974.

5. K. Löwner, Über monotone Matrixfunktionen, Math. Z. 38 (1934), 177-216.

6. M. Rosenblum and J. Rovnyak, Restrictions of analytic functions. I, Proc. Amer. Math. Soc. 48 (1975), 113-119.

7. Ju. L. Smul'jan, Monotone operator functions on a set consisting of an interval and a point, Ukrain. Mat. Ž. 17 (1965), no. 1, 130-136; English transl., Amer. Math. Soc. Transl. (2) 67 (1968), 25-32. MR 32 \#6239.

Graduate School of Arts and Sciences, University of Virginia, Charlottesville, VIRGINIA 22901 